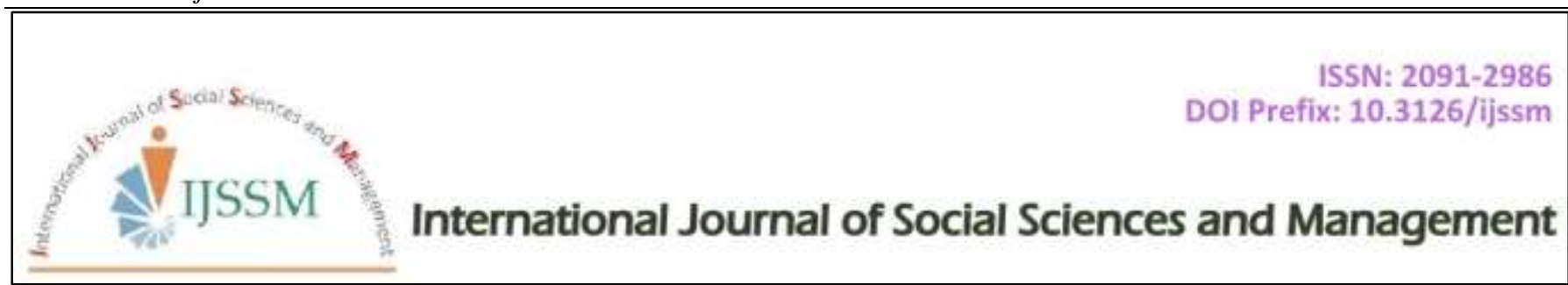

Research Article

\title{
Knowledge, Attitude and Practice of Blood Glucose Monitoring in Rural Area among Diabetic Patients
}

\author{
Saima Shabnum ${ }^{1 *}$, Hajra Sarwar ${ }^{1}$ \\ ${ }^{1}$ Lahore School of Nursing, The University of Lahore, Pakistan
}

\begin{abstract}
Background: Diabetes is main and growing health issue affecting more than 171 million peoples worldwide and the number is expected to rise to 366 million by 2030 . Type 2 Diabetes will keep on accounting for $90 \%$ of all the cases. According to the WHO, Pakistan positioned seventh in pervasiveness of Diabetes. In 2011, the assessed pervasiveness of diabetes in Pakistan was generally in excess of 350 million and it is depended upon to be in excess of 550 million by year 2030. In Pakistan 9.5\% of urban and $9.4 \%$ of the provincial population experience the bad effects of type 2 diabetes. Objective: The reason for this investigation was to survey learning, conduct in regards to blood glucose observing among diabetic in rural group, Lahore. Descriptive cross sectional investigation configuration was led to evaluate learning, disposition and routine with regards to blood glucose monitoring and a sample size of 100 participants was selected for this study through convenient sampling. Data was collected from the adult males and females of Husain Abad community. Result: The result show that there was low level of knowledge, somewhat positive attitudes but very low level of practices regarding the diabetic control and glucose monitoring among the participants. Conclusion: In conclusion, it is stated that this research study the knowledge of participants towards the diabetes was not good except the definition of diabetes. The attitude was comparatively positive and good for following different blood sugar controlling measures. The practices were very poor. No one was following regular exercises, dietary modifications etcetera.
\end{abstract}

Keywords: Knowledge Attitude; Practices Diabetes; Mellitus; Diabetic Control

\section{Introduction}

Diabetes, refers as diabetes mellitus, demonstrates a social event of metabolic issues in which the individual has high blood (glucose), either insulin age is insufficient, or the body's cells don't respond properly to insulin, or both (WHO, 2015). There are three standard kinds of diabetes these are Type 1, Type 2 and Gestational diabetes. Individuals with type 1 diabetes make by no or no insulin at all and it is called insulin subordinate. Sort 2 diabetes used to be called non-insulin subordinate diabetes or grown-up beginning diabetes, and records for no under $90 \%$ of all cases of diabetes. Gestational diabetes mellitus (GDM) is a kind of diabetes involving high blood glucose levels in the midst of pregnancy. Diabetes is an amazing and creating restorative issue impacting more than 171 million people worldwide and the number is depended upon to expanding to 366 million by 2030 . According to a survey did by the

\section{Cite this article as:}

S. Shabnum and H. Sarwar (2018) Int. J. Soc. Sc. Manage. Vol. 5, Issue-3: 225-230. DOI: 10.3126/ijssm.v5i3.20615

$1 *$ Corresponding author

Saima Shabnum,

Lahore School of Nursing, The University of Lahore, Pakistan

Email: saima.shabnum1278@gmail.com

Peer reviewed under authority of IJSSM

(C) 2018 International Journal of Social Sciences and Management

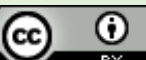

This is an open access article \& it is licensed under a Creative Commons Attribution 4.0 International License (https://creativecommons.org/licenses/by/4.0/) 
Diabetic Association of Pakistan recurrence of diabetes in Pakistan is around $10 \%$ (Shera et al., 2002).

In 2011, the assessed pervasiveness of diabetes in Pakistan was generally in excess of 350 million and it is depended upon to be in excess of 550 million by year 2030 (Ahmed $e t$ al., 2016). In Pakistan $9.5 \%$ of urban and $9.4 \%$ of the provincial population experience the bad effects of type 2 diabetes. Generally glucose intolerance (diabetes and hindered glucose resistance) is $22.04 \%$ in urban and $17.15 \%$ in provincial zones(Naqvi \& Kamal, 2013)Patient awareness about diabetes and its complications are very effective to control its negative impact on health. This knowledge can help the person to control and make plan related their daily life style according to status of the diabetes. Practices of blood glucose monitoring can prevent the further complications of DM, which affect altogether on the personal satisfaction of the diabetic patients. Data can enable individuals to evaluate their danger of diabetes, to persuade them to look for appropriate treatment and mind, and move them to assume responsibility of their malady for their lifetime. Diabetes instruction, with resulting change in learning, states of mind and abilities, prompts better control of the infection, and is broadly acknowledged to be a basic piece of far reaching diabetes mind despite the fact that the predominance of diabetes mellitus is high among populace in Middle East nations, patients regularly do not have the information and aptitudes to self-deal with their condition. The control of diabetes mellitus (DM) to a great extent relies upon patients' capacity to self-mind in their day by day lives, and thusly, understanding instruction is constantly viewed as basic component of DM management. The higher glycemic control diminishes the rate of intricacy and affirmation suggests that patients, who are capable about DM self-mind, have better long haul glycemic control component of DM administration. The enhanced glycemic control diminishes the rate of entanglements and confirmation proposes that patients, who are proficient about DM self-mind, have better long haul glycemic control..(Yazdanpanah, Nasiri, \& Adarvishi, 2015) The significance of this study is to give awareness through educational program about self-management of diabetes by self-monitoring and complication of diabetes to the rural community. Rural community have lack of knowledge regarding benefits of blood glucose monitoring and its complications if not manage properly. Moreover, these people have wrong practices due to which these are more prone to developing of complications. The prevalence of diabetes is very high in this rural community.

Research Question 1: What is the knowledge level in rural community has regarding blood glucose monitoring?
Research Question 2: what kind of attitude rural community possess toward blood glucose monitoring?

Research Question3: what are the practices of rural community have toward blood glucose monitoring and prevent from complications?

\section{Literature Review}

Reason behind the writing literature is to assess the proof and to take Knowledge about the point for the proposed project (White and Dudley-Brown, 2012). Diabetes is a vital and making helpful issue affecting in excess of 171 million individuals worldwide and the number is required to ascend to 366 million by 2030 . Sort 2 Diabetes will keep speaking to $90 \%$ of the broad number of cases. In Pakistan $9.5 \%$ of the urban and $9.4 \%$ of the country individuals experience the abhorrent effects of sort 2 diabetes. General glucose inclination (diabetes and crippled glucose flexibility) is $22.04 \%$ in urban and $17.15 \%$ in country areas. 2 According to the WHO measures, Pakistan arranged seventh in inevitability of Diabetes. These figures however address hint of a more critical test with different cases still new Knowledge is essential for patients (Gul, 2010).

Who don't think about reason for diabetes mellitus and its outcome few of the patients realize that one of general confusions of diabetes is kidney problem and coronary illness all in all. Inadequate knowledge of health problem and the significance of way of life adjustment is exceptionally evident among patients with diabetes. Absence of learning of diabetes mind among patients can effects affect their abilities to control diabetes. Coronary illness has been demonstrated as the most widely recognized complexity of diabetes by $72 \%$ of the patients. Consciousness of measures to recognize essential entanglements of diabetes, for example, circulatory strain observing, intermittent eye examination were poor, which uncovers the requirement for a few angles to be engaged in diabetes instruction programs. Awareness on way of life alteration with respect to weight gaining was low in this investigation. Some studies have demonstrated that advanced education was altogether connected with a superior awareness (Meng et al., 2011). Knowledge is a fundamental necessity for better consistence with restorative treatment (Tripathi, 2013). This cross-sectional investigation was completed to assess the information, disposition and works on with respect among 100 diabetes Iranians with 2 diabetics, going to the out-patient. Diabetes Clinic at Golestan Hospital, Ahvaz, Iran. A vis-à-vis meet was done and an approved Knowledge, attitude and the practice (KAP) survey was utilized to decide learning, Attitude and practice of diabetic patients. 53 percent of patients had great glycemic control. The outcomes demonstrated that mentality identified with diabetes was 
worthy in dominant part, while information and self-mind rehearses were lacking. The general mean $( \pm \mathrm{SD})$ score of information, demeanor and routine with regards to patients in light of KAP survey was medium level. There was no noteworthy relationship between the KAP score and $\mathrm{HbA1c}$ level (Mohammadi, Karim, Talib, and Amani, 2015).The cross sectional enlightening investigation for one year and directed at out-tolerant facilities of three family wellbeing focuses picked haphazardly in Dakahlia Governorate (Egypt). The general rate of satisfactory information with respect to diabetes was just $52.3 \%$ among members. Insulin-treated patients had most reduced learning, state of mind, and practice toward diabetes. There is a hole between patients' level of information and their practice. The examination was coordinated to diagram the general qualities, learning, mentality and practices of sort 2 diabetic patients embarking to the Out-Patient Department (OPD) of Baqai Institute of Diabology and Endocrinology (Karachi, Pakistan) amidst the period from July to September2000 from One hundred Type 2 diabetic patients Fifty-seven percent of the patients were Overweight or profound. Just $10.7 \%$ had remarkable glycemic control. Sixty seven percent did not do Exercise of any sort. The general care about the threat of complexity was elegant yet the misinformed judgments with respect to eating regimen, insulin and diabetes were exceptionally typical. A crosssectional overview and led in 2016, with respect to evaluating diabetes, socio-socioeconomics, related therapeutic conditions and self-mind in Endocrine Diabetic Clinic, Department of Medicine, Shaikh Zayed Hospital Lahore (Pakistan). The level of state of mind were likewise show as need be as low $8 \%$, medium $32 \%$ and high $60 \%$. ('Uthman, Ulla, and shah)

\section{Conceptual Framework}

Conceptual framework is given in Table 1.

\section{Methodology}

\section{Setting}

Health center of Hussain Abad community Lahore

\section{Research Design}

Correlational study design was used. As the objective the study was to. To assess the knowledge regarding blood glucose monitoring among adults in Hussain Abad community, Lahore. To assess the attitude regarding blood glucose monitoring among adults in Hussain Abad community, Lahore. To assess the practices about blood glucose monitoring and preventive complications among adults in Hussain Abad community, Lahore. Convenient sampling technique was used for the collection of data. This is non-probability sampling technique in which researcher selects subjects of his convenience and accessibility. Research instrument.

\section{Sampling}

The adopted questionnaire with some modification was used to assess the rural community Knowledge Attitude and Practice of Blood Glucose Monitoring among Diabetic's Patients Attending at community center. The permission will be taken from the related author of that questionnaire. The content validity of the questionnaire will be verified by the senior faculty of University of Lahore, The survey comprised of four areas Socio-statistic information, for example, age, sex, habitation, training, occupation, wage and so forth. Span of diabetes, and family history of diabetes. Patient's information around (11 questions) Causes of diabetes, hereditary factor, manifestations, typical level of blood glucose, reasons for hypoglycemia and its indications. State of mind of diabetic patients towards diabetes, consistence to recommended prescriptions feet mind, control of hypoglycemia and administration of diabetes amid disease (11 questions). Practice by diabetic patients will incorporate; wellspring of the gave social insurance administrations, recurrence of visits to diabetic facility,

Table 1: Conceptual framework

\section{Determinants}

- Knowledge

- Attitude

- Practice (Habit) - Quality of Life $\underline{\text { Health Outcomes }}$

- Self-management of Diabetes
Behavioral Problems

- Insufficient insight into Diabetes and its consequences

- Unsuccessful coping with

Diabetes

- Blood Glucose not Monitoring $\underline{\text { Health Problems }}$

- CVD diseases

- Kidney Disease

- Heart Problem

- Retinopathy

- Foot Ulcer 


\section{Methods Used to Analyze Data}

Data was analyzed by Statistical Package for Social Sciences (SPSS) version 21. Statistical analysis for study is Descriptive Statistics (frequency and percentage) for demographic characteristics. The study sample was analyzed by using measure of central tendency (mean, median, and mode). The descriptive data was about demographic data which include age, gender, education, income, family history. Dependent variables were knowledge, attitude and practice. Data was taken about the blood glucose monitoring form diabetic people in community.

\section{Study Timeline}

Lahore from September to December 2017

\section{Ethical Consideration}

At the authority level, official permission was taken from the principal or supervisor and co supervisor of University of Lahore to conduct the study. I obtained ethical clearance from Institutional Review Board (Kirby \& DiPaola). Permission was from the stakeholder of the community of Hussain Abad Lahore. At the household level, I explained the objectives of my study to the respondents and request verbal o written consent form the respondents. Data information were kept confidential. The participant has right to refuse to any answer to any question. Name of any respondents were not appeared in the venture. Earlier consent was taken from the establishment survey board (IRB) and the creators of evaluation devices. Educated assent of member was given with clear guideline. Members were educated about the reason and employments of the information gathering. Member protection and secrecy of data was guaranteed. The data gave by the member was utilized just for look into reason and not shared pointlessly. Regard for the respect of research members was organized. Member was permitted to pull back from inquire about whenever.

\section{Results and Discussion}

Results analysis of different components of the present study have Demographic data, Descriptive analysis of Knowledge, attitude and practice regarding Diabetes mellitus among the community people $40 \%$ and $30 \%$ respectively. The above table reveals that only $45 \%$ study participants had knowledge regarding the complications of Diabetic Mellitus, while 55\% were not aware of it. Similarly, $55 \%$ of the study participants had no knowledge that proper monitoring and control can prevent the complications of Diabetes. The knowledge of HBA 1-c was also very low among the participants and only 4o\% had knowledge about the test of HBA 1-C test.

\section{Demographic Data Analysis and Results}

Variables which are age, gender, marital status and family history of Diabetes, mellitus

Table 1: Frequency And Percentage Of Demographics Data

\begin{tabular}{lll}
\hline Age & $(\mathrm{N})$ & $\%$ \\
& & \\
$21-30$ years & 30 & $30 \%$ \\
$31-40$ years & 50 & $50 \%$ \\
$41-50$ years & 15 & $15 \%$ \\
Above 50 years & 5 & $5 \%$ \\
Gender & & \\
$\quad$ Male & 30 & $30 \%$ \\
Female & 70 & $70 \%$ \\
Marital Status & & \\
$\quad$ Single & 35 & $35 \%$ \\
Married & 65 & $65 \%$ \\
& & \\
Family History of DM & 25 & $25 \%$ \\
& 75 & $75 \%$ \\
\hline
\end{tabular}


Table 2: Respondents answers regarding diabetes.

\begin{tabular}{|c|c|c|c|c|}
\hline & $\mathrm{N}$ & $\%$ & $\mathrm{~N}$ & $\%$ \\
\hline What is diabetes? & 70 & $70 \%$ & 30 & $30 \%$ \\
\hline Risk factors of diabetes? & 40 & $40 \%$ & 60 & $06 \%$ \\
\hline Diabetic can be detected through. & 40 & $40 \%$ & 60 & $60 \%$ \\
\hline Frequency of diabetic patient measure her/his blood glucose level. & 35 & $35 \%$ & 65 & $65 \%$ \\
\hline What is hyperglycemic condition? & 40 & $40 \%$ & 60 & $60 \%$ \\
\hline What is hypoglycemic condition? & 30 & $30 \%$ & 70 & $70 \%$ \\
\hline Do you know the complications of diabetics, if not treated well? & 45 & $45 \%$ & 55 & $55 \%$ \\
\hline What type of life style modification do you think it helps for the control of diabetics? & 35 & $35 \%$ & 65 & $65 \%$ \\
\hline Monitoring of blood glucose level is important for reducing complication of diabetics. & 45 & $45 \%$ & 55 & $55 \%$ \\
\hline Do you know about hbA1-c? & 40 & $40 \%$ & 60 & $60 \%$ \\
\hline
\end{tabular}

Table 2 shows that $70 \%$ of the study participants knew about the definition of diabetes and while $30 \%$ did not have knowledge of what is diabetes. When the participants were asked about the risk factors of diabetes, the responses were that only $40 \%$ knew about the risk factors of diabetes while $60 \%$ did not know about it. The participants were also asked that how diabetes can be detected? in response, only $40 \%$ of the study participants had knowledge, while $60 \%$ were not having knowledge. on another question that how frequetly should a diabetic patient measure her/his blood glucose level, $35 \%$ of the study participants had knowledge, while $65 \%$ were not aware of this. of hyperglycemic and hypoglycemic conditions among the study participants was $40 \%$ and $30 \%$ respectively. The Table 2 reveals that only $45 \%$ study participants had knowledge regarding the complications of diabetic mellitus, while $55 \%$ were not aware of it. Similarly, $55 \%$ of the study participants had no knowledge that proper monitoring and control can prevent the complications of diabetes. The knowledge of hba 1-c was also very low among the participants and only $40 \%$ had knowledge about the test of hba 1-c test. During this study mostly the participants never any diabetic education programs and have low knowledge regarding complications and risk factors. Similarly previous studies also shows that awareness of measures to identify primary complications of diabetes, such as blood pressure monitoring, periodic eye examination were poor, which reveals the need for some aspects to be focused in diabetes education programs. the results of this study reveals that $20 \%$ of the participants disagreed, $15 \%$ remained neutral, $55 \%$ of the participants agreed and $10 \%$ strongly agreed that dietary modification can effectively control blood sugar level. Furthermore this study findings also reveals that $25 \%$ of the participants disagreed, $15 \%$ remained neutral, $50 \%$ of the participants agreed and $10 \%$ strongly agreed that every diabetic patient should do maximum to control their blood sugar level.

This examination additionally demonstrates that there was an uplifting mentality towards various measures with respect to diabetes. Past examinations likewise bolster the discoveries and results demonstrated that demeanor identified with diabetes was satisfactory in the lion's share, while learning and self-mind rehearses were inadequate. The general mean $( \pm \mathrm{SD})$ score of the information, state of mind and routine with regards to patients in light of KAP poll was in medium level. There was no huge relationship between the KAP scores and HbAlc level (Mohammadi, Karim, Talib, and Amani, 2015) In this study, the exercise is very low and $35 \%$ never do any exercise, $50 \%$ do it occasionally or rarely. Similarly by previous study only $10.7 \%$ had good glycemic control. Sixty seven percent did not do Exercise of such kind.The aims of this study were to assess knowledge, attitude and practices of blood glucose monitoring among diabetes in rural community, Lahore. The increased incidence of diabetic related complications e.g. retinopathy, kidney disease, and foot ulcer has been increased the economic burden on family and on government

\section{Limitations}

Limitations of the study were following Sample size $n=100$, which is low and cannot be generalized to the larger population. There was very short time to conduct this study, which can leads to some scientific and methodological mistakes 


\section{Conclusion}

In conclusion, it is stated that this research study the knowledge of participants towards the diabetes was not good except the definition of diabetes. The attitude was comparatively positive and good for following different blood sugar controlling measures. The practices were very poor. No one was following regular exercises, dietary modifications etcetera.

\section{Acknowledgement}

The first author wants to thank the accompanying individuals who from multiple points of view added to this bit of work. As a matter of first importance, she might wants to express gratitude toward the Principal of Lahore School of Nursing, Mr. Muhammad Afzal. She might wants to thank the subject personnel, Sir Afsar Ali for being her guider and for being the best motivation for the present work. Authors might likewise want to thank every who directly or indirectly helped during the study.

\section{References}

Ahmed MU, Seriwala HM, Danish SH, Khan AM, Hussain M, Husain M, Ahmed MM, Anis K. (2016) Knowledge, Attitude, and Self Care Practices Amongsts Patients WithType 2 Diabetes in Pakistan. Global journal of health science 8(7):1.

Gul N (2010) Knowledge, attitudes and practices of type 2 diabetic patients. Journal of Ayub Medical College Abbottabad 22(3):128-31.

Meng XJ, Dong GH, Wang D, Liu MM, Lin Q, Tian S, Xu LX, Hou H, Ren YF, Lee YL (2011) Prevalence, awareness, treatment, control, and risk factors associated with hypertension in urban adults from 33 communities of China: the CHPSNE study. Journal of hypertension 29(7): 1303-1310.

Mohammadi S, Karim NA, Talib RA, Amani R. (2015) Knowledge, attitude and practices on diabetes among type 2 diabetic patients in Iran: a crosssectional study. Science Journal of Public Health 3(4): 520-524.

Shera A. S, Jawad F, Basit A. (2002) Diabetes related knowledge, attitude and practices of family physicians in Pakistan. J Pak Med Assoc. 52

Tripathi KD (2013) Essentials of medical pharmacology. JP Medical Ltd.

White KM and Dudley-Brown S (2012) Translation of evidence for health policy. In K. M. White (Ed.), Translation of evidence into nursing and health care practice (pp. 131-148). New York, NY: Springer.

World Health Organization. (2015) The Selection and Use of Essential Medicines: Report of the WHO Expert Committee, 2015 (including the 19th WHO Model List of Essential Medicines and the 5th WHO Model List of Essential Medicines for Children). World Health Organization.

Yazdanpanah L, Nasiri M, Adarvishi S. (2015) Literature review on the management of diabetic foot ulcer. World journal of diabetes 6(1): 37. 